ARTICLE

\title{
Multiple acyl-CoA dehydrogenase deficiency kills Mycobacterium tuberculosis in vitro and during infection
}

\author{
Tiago Beites (10) ${ }^{1}$, Robert S. Jansen (10) 2,3, Ruojun Wang (1) 1,4, Adrian Jinich², Kyu Y. Rhee (1) 1,2, \\ Dirk Schnappinger ${ }^{1} \&$ Sabine Ehrt (1D ${ }^{1 \otimes}$
}

The human pathogen Mycobacterium tuberculosis depends on host fatty acids as a carbon source. However, fatty acid $\beta$-oxidation is mediated by redundant enzymes, which hampers the development of antitubercular drugs targeting this pathway. Here, we show that rv0338c, which we refer to as etf $D$, encodes a membrane oxidoreductase essential for $\beta$-oxidation in $M$. tuberculosis. An etfD deletion mutant is incapable of growing on fatty acids or cholesterol, with long-chain fatty acids being bactericidal, and fails to grow and survive in mice. Analysis of the mutant's metabolome reveals a block in $\beta$-oxidation at the step catalyzed by acyl-CoA dehydrogenases (ACADs), which in other organisms are functionally dependent on an electron transfer flavoprotein (ETF) and its cognate oxidoreductase. We use immunoprecipitation to show that $M$. tuberculosis EtfD interacts with FixA (EtfB), a protein that is homologous to the human ETF subunit $\beta$ and is encoded in an operon with fixB, encoding a homologue of human ETF subunit $\alpha$. We thus refer to FixA and FixB as EtfB and EtfA, respectively. Our results indicate that EtfBA and EtfD (which is not homologous to human EtfD) function as the ETF and oxidoreductase for $\beta$-oxidation in $M$. tuberculosis and support this pathway as a potential target for tuberculosis drug development.

\footnotetext{
${ }^{1}$ Department of Microbiology and Immunology, Weill Cornell Medical College, New York, NY 10065, USA. ${ }^{2}$ Division of Infectious Diseases, Department of Medicine, Weill Cornell Medical College, New York, NY 10065, USA. ${ }^{3}$ Present address: Department of Microbiology, Radboud University, 6525 AJ Nijmegen, The Netherlands. ${ }^{4}$ Present address: Department of Molecular Biology, Princeton University, Princeton, NJ 08540, USA.

$\varpi_{\text {email: sae2004@med.cornell.edu }}$
} 
aintenance of an energized membrane is essential for Mycobacterium tuberculosis (Mtb) to grow and survive periods of non-replicating persistence ${ }^{1}$. This need has driven tuberculosis (TB) drug development efforts towards Mtb's energy metabolism. These efforts are supported by the first antiTB drug approved in over 40 years-the ATP synthase inhibitor bedaquiline ${ }^{2}$.

Mtb's energy related pathways exhibit varying degrees of vulnerability to inhibition. Uptake of its main carbon sources in vivo is performed by specialized transporters: the multi-subunit Mce1 complex transports fatty acids ${ }^{3}$ and the Mce 4 complex facilitates the uptake of cholesterol ${ }^{4}$. Inactivation of Mcel can reduce intracellular growth $^{5}$, although it has also been reported to cause hypervirulence ${ }^{6}$, while Mce4 was conclusively shown to be essential for survival during the chronic phase of infection ${ }^{4}$. LucA, which acts as a regulator of both Mcel and Mce4, is also required for wild type levels of Mtb virulence ${ }^{3}$, further supporting that inhibiting the ability to import host lipids affects Mtb's pathogenicity.

Cholesterol degradation yields multiple products, including acetyl-CoA, propionyl-CoA, pyruvate, and likely succinyl-CoA that can be used for energy generation or lipid biosynthesis ${ }^{7-11}$. Deletion of genes encoding cholesterol oxidation enzymes attenuated growth ${ }^{12}$ and caused survival defects of Mtb in mice ${ }^{13,14}$. A screen against Mtb residing in the phagosomes of macrophages identified several compounds that target cholesterol metabolism ${ }^{15}$. In contrast, fatty acids are degraded solely through $\beta$-oxidation. Mtb's genome encodes multiple enzymes for each step of $\beta$-oxidation, including 34 putative acyl-CoA ligases, 35 putative acyl-CoA dehydrogenases, 22 putative enoyl-CoA dehydratase, five putative $\beta$-hydroxyacyl-CoA dehydrogenase, and six putative thiolases ${ }^{16}$. Some of these enzymes are necessary for infection, but they also have been shown to play roles in other pathways, such as complex lipid biosynthesis ${ }^{17}$ and cholesterol degradation ${ }^{18}$. Due to the apparent redundancy of Mtb's fatty acid $\beta$-oxidation machinery, this metabolic pathway has thus been presumed to be invulnerable to chemical inhibition.

In the present study, we define an enzyme complex that has previously not been recognized as required for fatty acid degradation in Mtb. It consists of an electron transfer flavoprotein composed by two subunits-FixA (Rv3029c) and FixB (Rv3028c) - and a membrane oxidoreductase (Rv0338c), which we propose to re-name as $\mathrm{EtfB}_{\mathrm{Mtb}}$, $\mathrm{EtfA}_{\mathrm{Mtb}}$, and $\mathrm{EtfD}_{\mathrm{Mtb}}$ based on their human counterparts. Deletion of Mtb's EtfD causes multiple acylCoA dehydrogenase deficiency, which prevents utilization of fatty acids as carbon sources and can kill $\mathrm{Mtb}$ in vitro and during mouse infection.

\section{Results}

A possible role for EtfD $_{M t b}$ in Mtb's energy metabolism. $\mathrm{EtfD}_{\mathrm{Mtb}}(\mathrm{Rv0338})$ is a membrane protein of unknown function predicted to be essential for growth of Mtb on agar plates ${ }^{19,20}$. To experimentally determine its topology, we fused E. coli alkaline phosphatase PhoA to EtfD at specific residues of the predicted transmembrane helices. Transport of PhoA outside of the cytoplasm enables reactivity with the chromogenic substrate 5bromo-4-chloro-3-indolyl phosphate p-toluidine (BCIP). Based on this assay, the soluble portion of $\mathrm{EtfD}_{\mathrm{Mtb}}$ faces the cytoplasm (Supplementary Fig. 1a), and this topology agrees with the prediction generated by the MEMSAT3 algorithm ${ }^{21}$ (Supplementary Fig. 1b).

Next, we performed in silico analysis, which placed $\mathrm{EtfD}_{\mathrm{Mtb}}$ into the cluster of orthologue groups 0247 (COG0247) composed of $\mathrm{Fe}-\mathrm{S}$ oxidoreductases involved in energy production and conversion (Fig. 1a). COG0247 includes a variety of enzymes, including lactate dehydrogenase and the methanogenic-related heterodisulfide reductase; however, most proteins in COG0247 remain uncharacterized and are of unknown function. EtfD $\mathrm{Mtb}_{\mathrm{tb}}$ is predicted to be a chimeric enzyme assembled from four domains: an N-terminal domain, which is similar to the gamma subunit of nitrate reductases and putatively binds a cytochrome b; a central $4 \mathrm{Fe}-4 \mathrm{~S}$ di-cluster domain similar to succinate dehydrogenases; and two C-terminal cysteine-rich domains (CCG), which are often found in heterodisulfide reductases (Fig. 1b). This analysis led us to hypothesize that EtfD is a component of Mtb's energy metabolism.

EtfD $_{M t b}$ is linked to fatty acid metabolism and it is essential in vivo. To analyze the impact of $\mathrm{EtfD}_{\mathrm{Mtb}}$ depletion on Mtb's growth in vitro, we generated a TetOFF strain, in which EtfD levels are controlled by anhydrotetracycline (ATC)-inducible proteolysis ${ }^{22}$. Depletion of $\mathrm{EtfD}_{\mathrm{Mtb}}$ inhibited Mtb's growth in regular medium supplemented with oleic acid, albumin, dextrose and catalase (OADC), which is consistent with its predicted essentiality (Fig. 1c, d). Curiously, when the same medium was supplemented with a fatty acid free enrichment (albumin, dextrose and sodium chloride, $\mathrm{ADN}$ ), depletion of $\mathrm{EtfD}_{\mathrm{Mtb}}$ did not impact growth to the same extent (Fig. 1c, d). This is similar to the fatty acid sensitive phenotype observed in response to inactivation of Mtb's type II NADH dehydrogenases ${ }^{23}$, which allowed the genetic deletion of Ndh-2 by growth in a fatty acid free medium. We applied the same strategy to $\operatorname{etf} D_{\mathrm{Mtb}}$ and generated a deletion strain $(\Delta e t f D)$ (Supplementary Fig. 2a). The $\Delta$ etfD strain was confirmed by whole genome sequencing (WGS) to not contain additional polymorphisms known to affect growth (Supplementary Table 1). This knockout strain also phenocopied the fatty acid sensitivity observed with the knockdown mutant (Supplementary Fig. 2b, c).

We next sought to investigate the importance of etf $D_{\mathrm{Mtb}}$ for pathogenesis in an aerosol model of TB infection in mice. After aerosol infection, $\Delta e t f D$ was unable to grow in mouse lungs and declined in viability from day 14 onwards (Fig. 2a and Supplementary Fig. 3a). In agreement with the CFU data, gross lung pathology showed no lesions in the mice infected with $\Delta$ etfD (Fig. 2b and Supplementary Fig. 3b). The attenuation was even more pronounced in spleens, where no $\Delta$ etfD CFU were recovered at any time point (Fig. 2a and Supplementary Fig. 3a). All phenotypes were rescued by reintroducing an intact copy of etfD $D_{\mathrm{Mtb}}$.

Mtb requires $\mathrm{EtfD}_{\mathrm{Mtb}}$ to consume fatty acids and cholesterol as a carbon source and to prevent toxicity of long-chain fatty acids. The fatty acid sensitivity of $\Delta$ etfD suggested that this protein is, directly or indirectly, required for fatty acid metabolism. To test this further, we grew strains in media with different single carbon sources. $\triangle e t f D$ was able to grow in both glycolytic (glycerol) and gluconeogenic (acetic acid and propionic acid) carbon sources, although at a slower rate than the wild type (Fig. 3a). However, longer chain fatty acids (butyric acid, palmitic acid and oleic acid) did not support detectable growth of $\Delta e t f D$ (Fig. 3b). Fatty acids with four carbons or more in length require functional $\beta$-oxidation to be utilized, hence these results indicated that $\Delta e t f D$ might display a defect in $\beta$-oxidation. Mtb oxidizes the cholesterol side-chain through $\beta$-oxidation. Enzymes involved in cholesterol side-chain degradation are essential for Mtb to grow with cholesterol as single carbon source ${ }^{7,12}$. Similarly, $\Delta$ etfD was not able to grow with cholesterol as a single carbon source (Fig. 3c).

To distinguish between two possible interpretations, namely 1) $\Delta e t f D$ simply failed to use fatty acids as carbon source and 2) $\Delta e t f D$ was intoxicated by fatty acids, we tested if glycerol could 

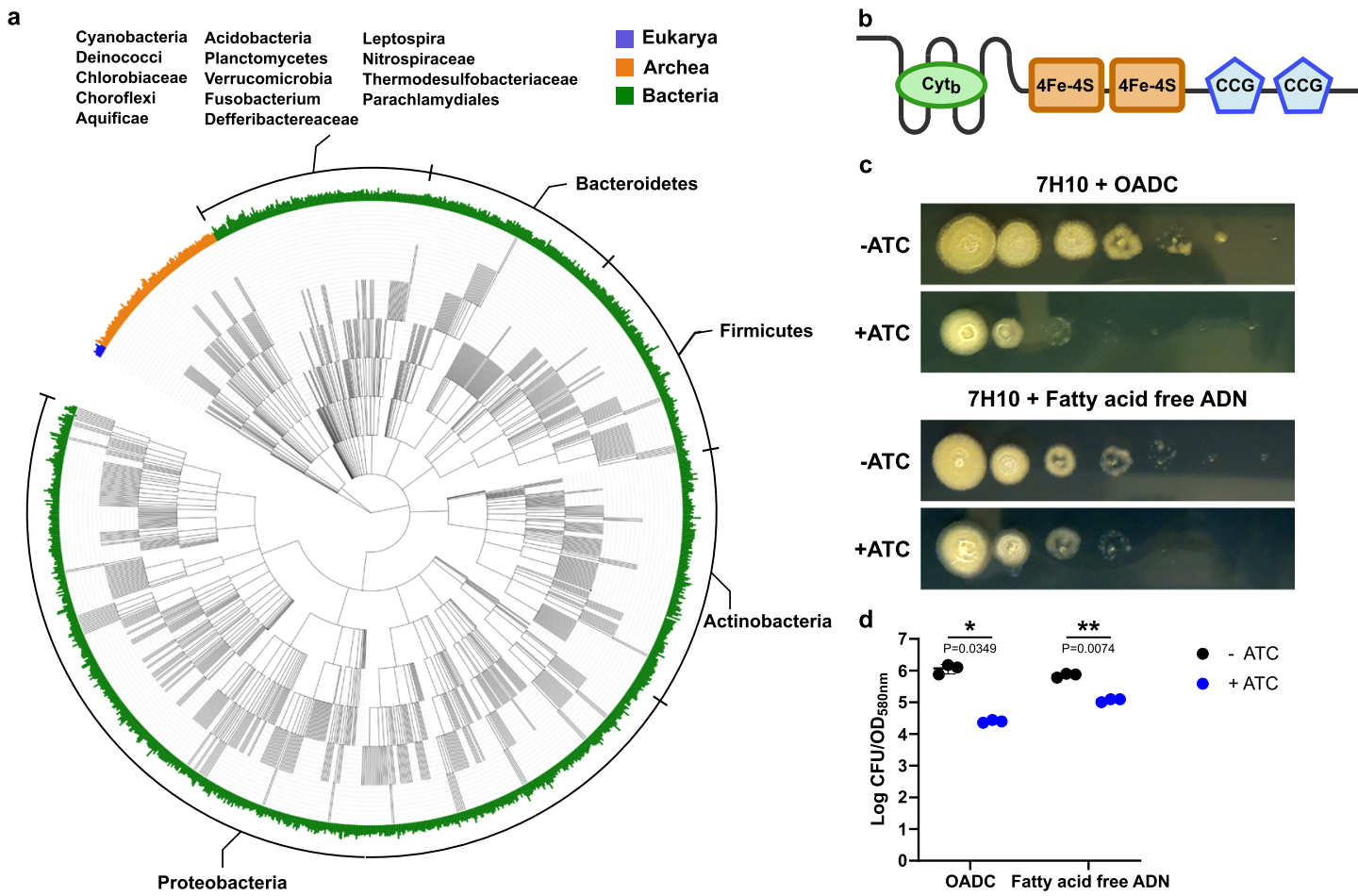

Fig. 1 EtfD is a membrane protein possibly involved in Mtb's energy metabolism. a Rootless phylogenetic tree with species containing members of COG0247 according to the database of the webtool EggNog. The inner colored ring corresponds to life domains, while the outer ring refers to bacteria phyla. b Domain architecture of EtfD based on the algorithms of HHPred and Xtalpred. Cyt ${ }_{b}$ cytochrome b, CCG cysteine-rich domain. $\mathbf{c}$ Spot assay on solid media with an EtfD-TetOFF strain, where protein levels are controlled by anhydrotetracycline (ATC). Serial dilutions (106 down to 1 bacteria) were incubated for 14 days. These results are representative of three independent experiments. $\mathbf{d}$ Quantification of spot assays (c). This quantification aggregates the data from the three independent experiments. To quantify the spot assays, we picked the first dilution where isolated CFUs could be counted and divided it by the optical density (OD) of the corresponding bacterial suspension. The discrepancy between the OD of the bacterial suspension and the corresponding CFUs, which is due to sensitivity to lipids in the solid media, is reflected by this ratio. Data are averages with individual data points depicted. Error bars correspond to standard deviation. Statistical significance was assessed through paired two-tailed $t$-test. ${ }^{\star} P<0.05 ;{ }^{\star \star} P<0.01$.
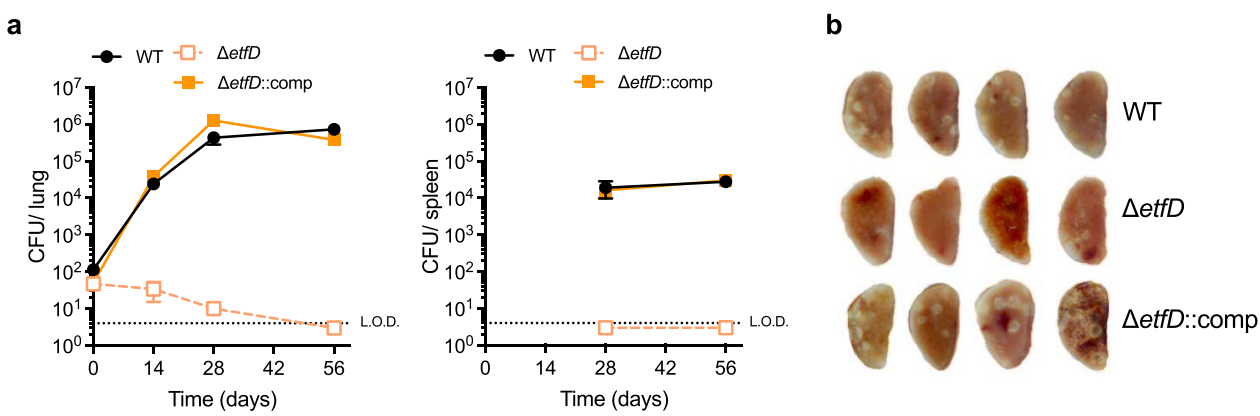

Fig. 2 EtfD is essential for growth and survival in vivo. a Growth and persistence of wild type Mtb, $\Delta$ etfD and the complemented mutant in mouse lungs and spleens. Data are CFU averages from four mice per time point and are representative of two independent experiments. Error bars correspond to standard deviation. "Comp" stands for complemented. L.O.D. stands for limit of detection. b Gross pathology of lungs infected with wild-type Mtb, $\Delta$ etfD and the complemented mutant at day 56.

rescue the impaired growth of $\Delta$ etfD with fatty acids. Glycerol was able to restore growth in medium with butyric acid as carbon source, but it was not able to restore growth with long-chain fatty acids. Cholesterol partially inhibited growth in this mixed carbon source setup (Supplementary Fig. 4). This argued for a toxic effect and led us to evaluate the impact of fatty acids and cholesterol on the viability of $\triangle e t f D$. We found that butyric acid and cholesterol were not toxic, while long-chain fatty acids were bactericidal to $\Delta$ etfD (Fig. 3d). Glycerol did not rescue the bactericidal effect of long-chain fatty acids, and partially rescued growth with cholesterol in the medium (Fig. 3d and Supplementary Fig. 4).
Mtb acyl-CoA dehydrogenase activity requires $\mathrm{EtfD}_{\mathrm{Mtb}}$. We applied metabolomics to understand why the consumption of fatty acids is prevented in the absence of $\mathrm{EtfD}_{\mathrm{Mtb}}$. We focused these studies on ${ }^{13} \mathrm{C}_{4}$-labeled butyric acid to isolate the impact of etfD disruption on fatty acid consumption from potentially additional confounding effects associated with the toxicity of longer chain fatty acids. Inspection of labelled metabolites in central carbon metabolism confirmed the presence of butyric acid in WT, $\Delta e t f D$, and the complemented mutant (Fig. 4 and Supplementary Fig. 5). All three strains were thus able to import ${ }^{13} \mathrm{C}_{4}$-labelled butyric acid. 


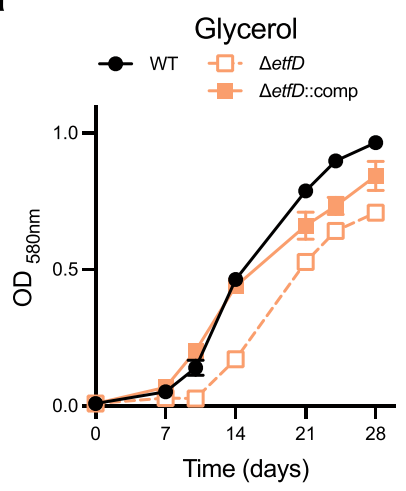

b

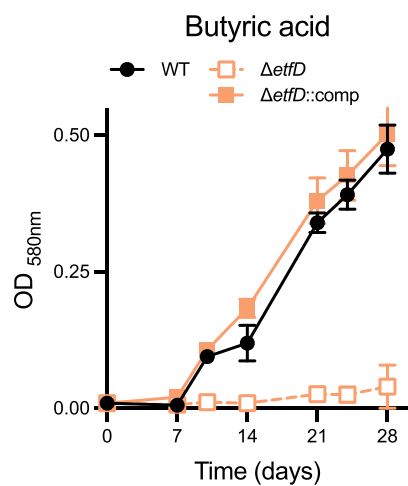

c

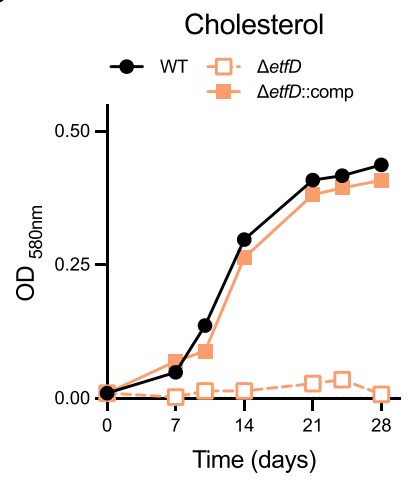

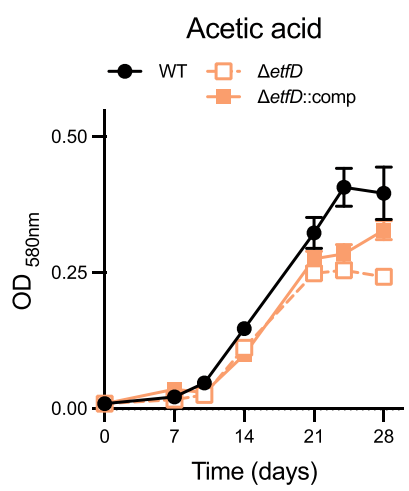
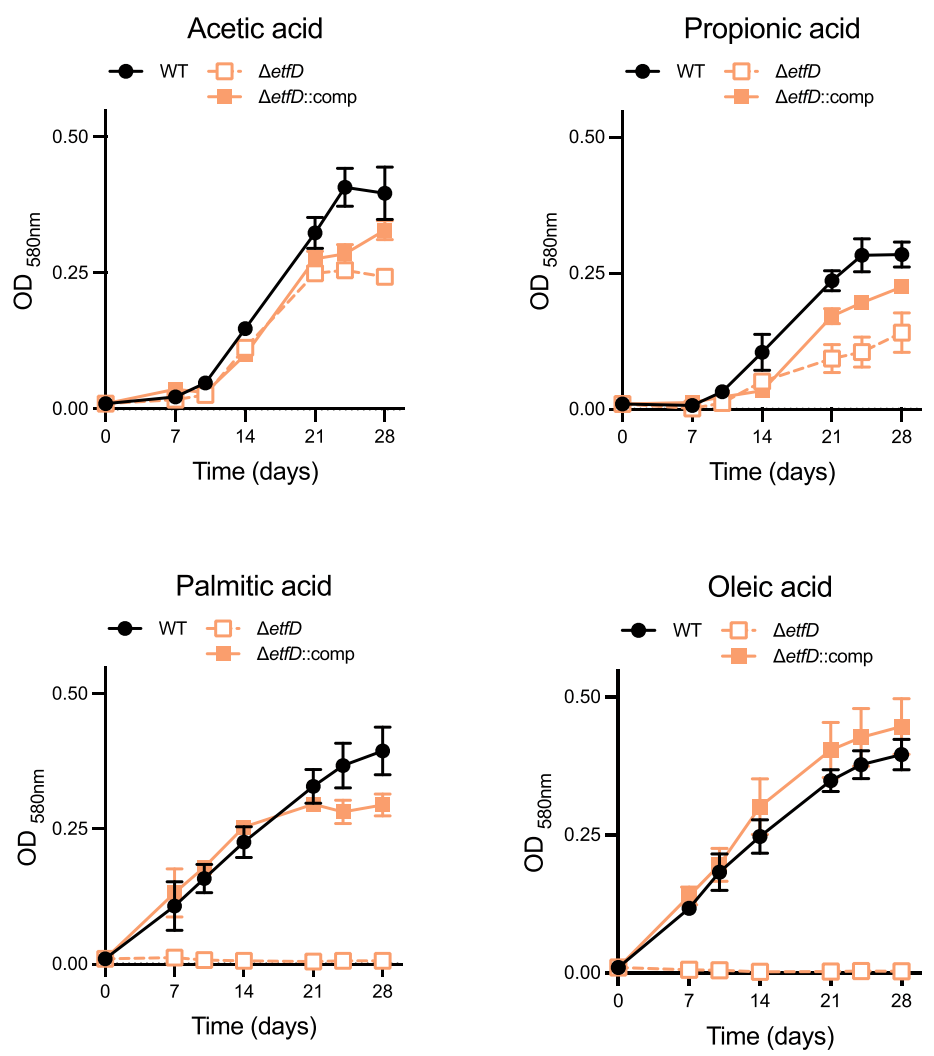

d

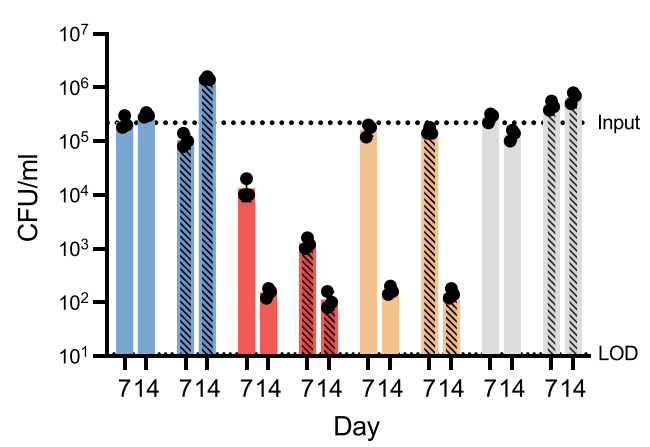

Butyric acid

N Butyric acid + Glycerol

Palmitic acid

N Palmitic acid + Glycerol

Oleic acid

N Oleic acid + Glycerol

Cholesterol

N Cholesterol + Glycerol

Fig. 3 EtfD is necessary for the utilization of fatty acids that require $\boldsymbol{\beta}$-oxidation. a-c Strains were grown in media with single carbon sources that a do not require and $\mathbf{b}$ require $\beta$-oxidation for catabolism, as well as cholesterol (c). Carbon sources were used at the following final concentrations: glycerol $25 \mathrm{mM}$, acetic acid $25 \mathrm{mM}$, propionic acid $2.5 \mathrm{mM}$, butyric acid $2.5 \mathrm{mM}$, palmitic acid $250 \mu \mathrm{M}$, oleic acid $250 \mu \mathrm{M}$, and cholesterol $250 \mu \mathrm{M}$. To sustain growth and avoid toxicity palmitic acid and oleic acid were replenished every 3-4 days for the first 14 days of culture. Cholesterol was replenished every to 3 to 4 days for the first 14 days to minimize precipitation. Data are averages of three replicates and are representative of three independent experiments. Error bars correspond to standard deviation. "Comp" stands for complemented. d Viability of $\Delta$ etfD in media with single carbon sources (butyric acid, palmitic acid, and oleic acid), or in mixed carbon sources (fatty acids and glycerol) at the same concentrations used in $\mathbf{a}$ and $\mathbf{b}$ was assessed at days 7 and 14. Data are averages of three replicates and are representative of three independent experiments. Error bars correspond to standard deviation. "Comp" stands for complemented. LOD stands for limit of detection.

To be catabolized through $\beta$-oxidation, butyric acid needs to be transformed by an acyl-CoA ligase into butyryl-CoA. Strikingly, butyryl-CoA accumulated in $\triangle$ etfD approximately 45 -fold relative to $\mathrm{WT}$ and the complemented mutant. The remaining intermediates of butyric acid $\beta$-oxidation were not detectable in any of the strains, but the pool size of the end-product acetyl-CoA was approximately 2 -fold lower in $\Delta$ etfD than in WT. Labelled TCA cycle intermediates, with the exception of succinyl-CoA, were also partially depleted in $\Delta e t f D$ (Fig. 4 and Supplementary Fig. 5). The metabolomic profile of $\triangle e t f D$, specifically the accumulation of butyryl-CoA, strongly suggested that inactivation of EtfD interfered with the function of acyl-CoA dehydrogenases (ACAD), which in turn impaired fatty acid catabolism.

EtfD $_{M t b}$ interacts with an electron transfer protein. We immunoprecipitated $\mathrm{EtfD}_{\mathrm{Mtb}}$ and identified putative interacting proteins by mass spectrometry. Among the 49 total hits (Supplementary Table 2), 41 were located or predicted to be located at the membrane/cell wall, while the remaining eight were cytoplasmic proteins. To investigate possible links to $\beta$-oxidation-a cytoplasmic process-we focused on the cytoplasmic interactors, 


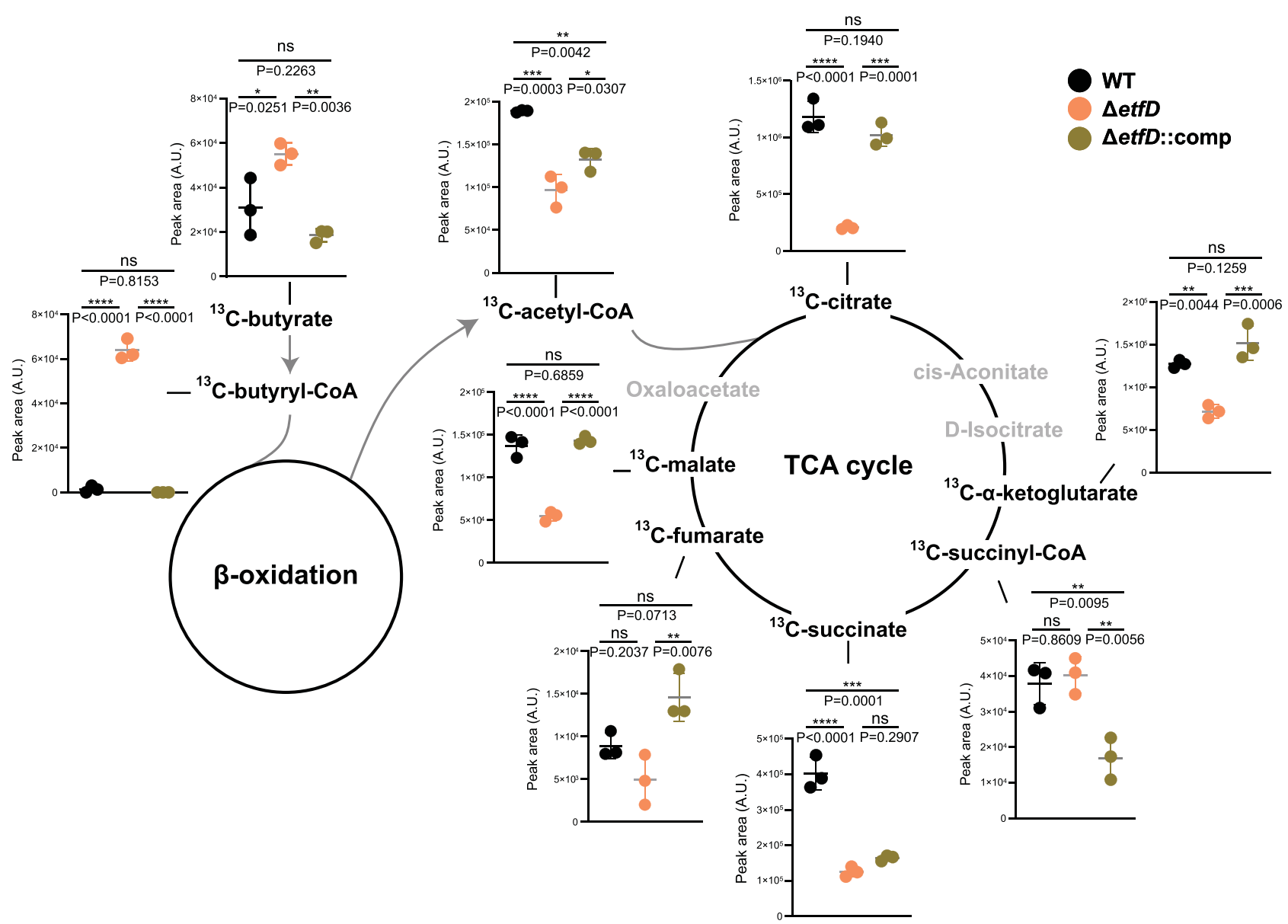

Fig. 4 Stable isotope tracing reveals a block in $\beta$-oxidation at the level of acyl-CoA dehydrogenases. Strains were grown on filters on top of solid medium permissible to $\Delta$ etfD growth for 7 days and then transferred to solid media with ${ }^{13} \mathrm{C}_{4}$-labelled butyric acid $(2.5 \mathrm{mM})$ as single carbon source for $24 \mathrm{hrs}$. Levels of the indicated ${ }^{13} \mathrm{C}$-labeled metabolites (total ${ }^{13} \mathrm{C}$ pool, except for ${ }^{13} \mathrm{C}_{4}$-butyrate) were quantified by LC-MS analysis. Data are averages of three replicates and are representative of two independent experiments. Error bars correspond to standard deviation. "Comp" stands for complemented. Statistical significance was assessed by one-way ANOVA followed by post hoc test (Tukey test; GraphPad Prism). ${ }^{\star} P<0.05 ;{ }^{\star \star} P<0.01$; ${ }^{\star \star \star} P<0.001$; ${ }^{\star \star \star \star} P<0.0001$. ns not significant. \# metabolites with no statistically significant difference between wild-type and $\Delta$ etfD in the second independent experiment.

which consisted of two flavoproteins $\mathrm{EtfB}_{\mathrm{Mtb}}$ and Rv1279, the sigma factor SigA, a putative helicase Rv1179c, an exopolyphosphatase Ppx1, the phthiocerol dimycocerosate (PDIM) biosynthesis enzyme $\mathrm{PpsC}$, the protease $\mathrm{ClpP} 2$, and $\mathrm{Rv} 1215 \mathrm{c}$, a protein with putative proteolytic activity (Fig. 5a). We were especially interested in the flavoprotein $\mathrm{EtfB}_{\mathrm{Mtb}}$, because of its homology (30\% identity; $87 \%$ coverage) with the beta-subunit of the human electron transfer flavoprotein (ETF). Moreover, etf $B_{\mathrm{Mtb}}$ (annotated as fixA-rv3029c) forms an operon with etf $A_{\mathrm{Mtb}}$ (annotated as $f(x B-r v 3028 c$ ), which shares homology with the alpha subunit of the human ETF (41\% identity: $98 \%$ coverage). In humans, $\mathrm{ETF}^{24}$ interacts with a cognate membrane oxidoreductase $^{25}$ (EtfD) and both are required to re-oxidize the FAD co-factor of multiple ACADs. This led us to hypothesize that Mtb might display a similar activity. Although $\mathrm{EtfD}_{\mathrm{Mtb}}$ is not a homolog of the human EtfD, our working model predicted that EtfBA $_{\mathrm{Mtb}}$ and $\mathrm{EtfD}_{\mathrm{Mtb}}$ constitute a complex necessary for the activity of ACADs in Mtb (Fig. 5b).

To further assess a functional connection between $\mathrm{EtfD}_{\mathrm{Mtb}}$ and EtfBA $_{\mathrm{Mtb}}$, we asked if these proteins co-occur across bacterial proteomes. A BLASTp search against a database of 6240 bacterial proteomes (identity cutoff of $>30 \%$ and coverage cutoff of $>75 \%$ ) identified $469 \mathrm{EtfD}_{\mathrm{Mtb}}, 473 \mathrm{EtfB}_{\mathrm{Mtb}}$, and $472 \mathrm{EtfA}_{\mathrm{Mtb}}$ homologs, $98 \%$ of which occur in actinobacteria, with the spirochete
Leptospira interrogans-the causative agent of leptospirosis-as a notable exception (Fig. 5; and Supplementary Data 1). EtfD, EtfB and EtfA showed a strong co-occurrence $\left(P\right.$-value $\left.<10^{-10}\right)$, which was suggestive of a functional connection. Curiously, similar to $\mathrm{Mtb}, \mathrm{L}$. interrogans is proposed to use host-derived fatty acids as the primary carbon sources during infection ${ }^{26}$.

These results support the hypothesis that EtfD $_{\mathrm{Mtb}}$ serves as oxidoreductase for EtfBA $\mathrm{Mtb}_{\mathrm{tb}}$, which in turn is necessary for the activity of ACADs.

EtfBA $_{M t b}$ and EtfD $_{M t b}$ are required for acyl-CoA dehydrogenase activity. If $\mathrm{EtfBA}_{\mathrm{Mtb}}$ and $\mathrm{EtfD}_{\mathrm{Mtb}}$ participate in the same biochemical pathway then inactivation of $\mathrm{EtfBA}_{\mathrm{Mtb}}$ should also impair the use of fatty acids and cholesterol as single carbon sources by Mtb. To test this prediction, we first isolated a knockout strain for etfBA in fatty acid free medium (Supplementary Fig. 6) and confirmed its genetic identity through WGS (Supplementary Table 1). We then grew wild type, $\triangle e t f B A$ and complemented $\triangle e t f B A$ in media with different carbon sources. $\triangle$ etfBA was able to grow with glycerol, albeit slower than WT (Fig. 6a). In contrast, we did not detect growth in butyric acid, oleic acid, or cholesterol as single carbon sources, corroborating our prediction (Fig. $6 \mathrm{~b}-\mathrm{d}$ ). 
a

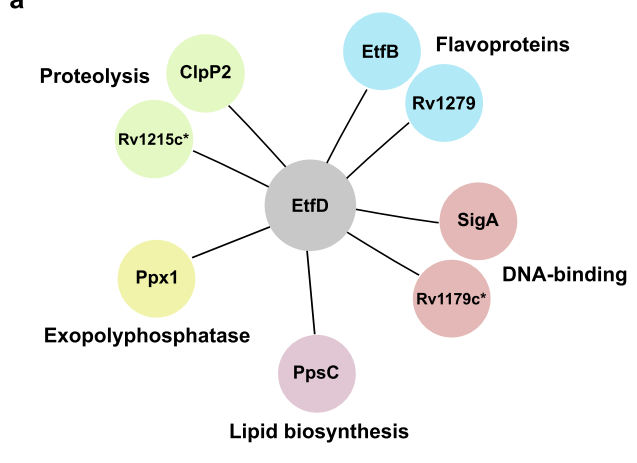

c

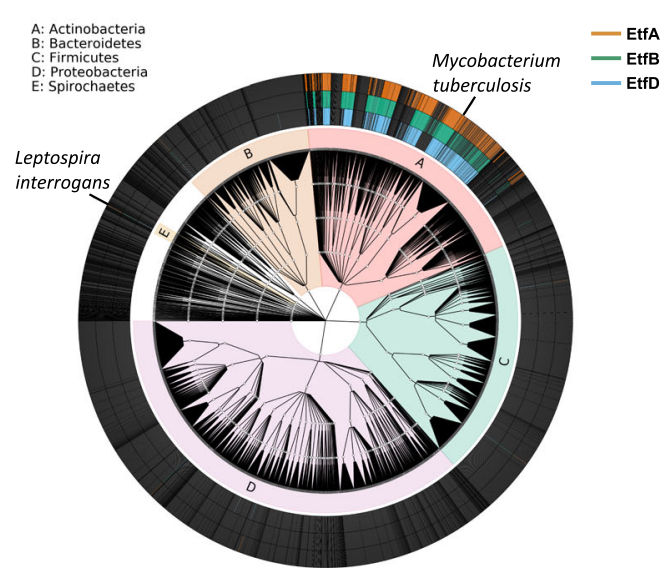

b

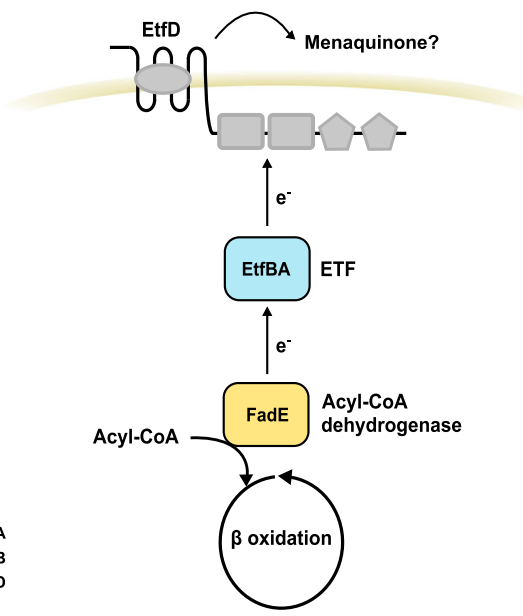

Fig. 5 EtfD and EtfBA interaction and co-occurrence. a EtfD cytoplasmic interactors identified by protein co-immunoprecipitation. $\mathbf{b}$ Model for the pathway constituted by EtfD and EtfBA. c Uniprot bacterial proteome database was surveyed for EtfD and EtfBA putative homologs. The inner ring corresponds to Phyla and the outer rings represent strains with a hit ( $>30 \%$ identity and $>75 \%$ coverage) for EtfD, EtfB, or EtfA. Asterisk $\left({ }^{*}\right)$ in silico prediction.

To independently test the role of $\mathrm{EtfBA}_{\mathrm{Mtb}}$ and $\mathrm{EtfD}_{\mathrm{Mtb}}$ in $\beta$ oxidation, we assessed if an acyl-CoA oxidase (ACO) could allow both $\triangle e t f B A$ and $\triangle e t f D$ to grow with fatty acids. ACOs are peroxisomal enzymes that catalyze the same reaction as ACADs using molecular oxygen to re-oxidize FAD rather than ETF/ ETFD $^{27}$. The gene pox3, which encodes the extensively characterized ACO Pox3 from the yeast Yarrowia lipolytica ${ }^{28,29}$ (Fig. 6d), was codon adapted for Mtb and expressed under the control of a strong, constitutive promoter in both $\triangle e t f B A$ and $\Delta e t f D$. Pox3 has a high affinity for fatty acids with six and eight carbons chain length and low affinity for most other fatty acids ${ }^{28}$; thus, we grew strains in a medium with octanoic acid (high affinity), butyric acid (low affinity), or oleic acid (low affinity) as single carbon sources. $\Delta e t f D$ was not able to utilize octanoic acid as single carbon source, further confirming its inability to oxidize multiple fatty acids, while the growth rate of $\Delta$ etfD::pox3 was similar to that of wild type and complemented strain (Fig. 6e). A similar result was obtained with butyric acid, although it took longer for $\Delta$ etfD::pox3 to reach wild type density (Supplementary Fig. 7). As expected, based on the affinity profile and function of Pox3, pox3 did not rescue $\Delta$ etfD growth with oleic acid as single carbon source and it did not alter the growth rate on glycerol. Similarly, $\triangle$ etf $B A$ did not grow on octanoic acid as single carbon source, while $\triangle$ etfBA::pox3 was able to grow in the same medium, although reaching a lower final optical density than WT and complemented mutant. $\triangle e t f B A:: p o x 3$ was able to grow with butyric acid entering stationary phase 21 days after wild-type and complemented strain, it did not grow with oleic acid and it conferred a slight advantage on glycerol when compared with $\triangle$ etfBA (Fig. 6e and Supplementary Fig. 7). Acyl-CoA oxidase activity was confirmed in the cell lysates of Pox3 expressing strains (Supplementary Fig. 8).

These results confirmed that EtfBA $\mathrm{Mtb}_{\mathrm{tb}}$ and $\mathrm{EtfD}_{\mathrm{Mtb}}$ constitute a complex necessary for the activity of fatty acid $\beta$-oxidation ACADs.

\section{Discussion}

Mtb's energy metabolism displays a remarkable plasticity which supports its adaptation to a multitude of host microenvironments ${ }^{30}$. Its unusual domain structure suggested that the membrane protein encoded by $r v 0338 \mathrm{c}$ could be an unrecognized, essential component of Mtb's energy metabolism. Our studies identified Rv0338c as a member of a short electron transfer pathway essential for ACADs activity. Based on the similarity of this system to the human ETF system we propose to rename the respective $\mathrm{Mtb}$ genes as etf $D_{\mathrm{Mtb}}(r v 0338 c)$, etf $B_{\mathrm{Mtb}}$ (rv3028c), and etf $A_{\mathrm{Mtb}}(r v 3029 c)$.

The increased susceptibility to fatty acids of an EtfD $_{\mathrm{Mtb}}$ TetOff strain suggested a possible connection with fatty acid metabolism. Accordingly, our data showed that an etf $D_{\mathrm{Mtb}}$ deletion mutant was not capable of utilizing fatty acids with four carbons or more as single carbon sources, thus indicating impairment in $\beta$ oxidation. We also found that $\Delta$ etfD cannot utilize cholesterol. These metabolic defects were associated with different outcomes regarding viability: butyric acid (short-chain) was not toxic, while palmitic acid and oleic acid (long-chain) were bactericidal with or without glycerol and cholesterol negatively impacted growth in mixed carbon source medium (glycerol + cholesterol). Importantly, $\Delta e t f D$ did not grow and presented a survival defect in mice. Long-chain fatty acids, including oleic acid, are common 
a

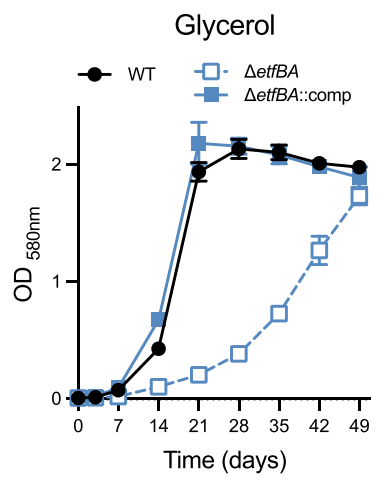

d

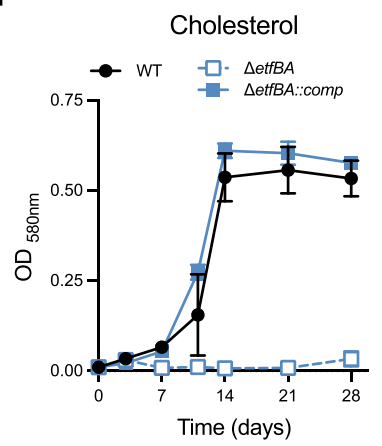

b

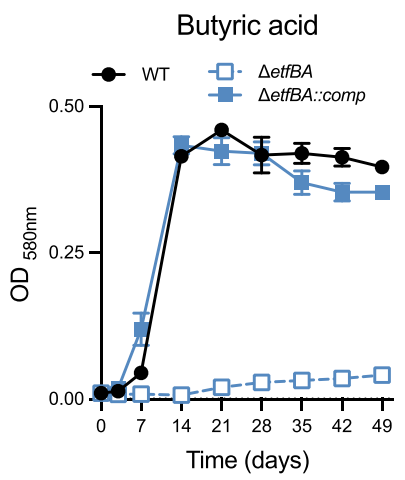

$\mathbf{e}$

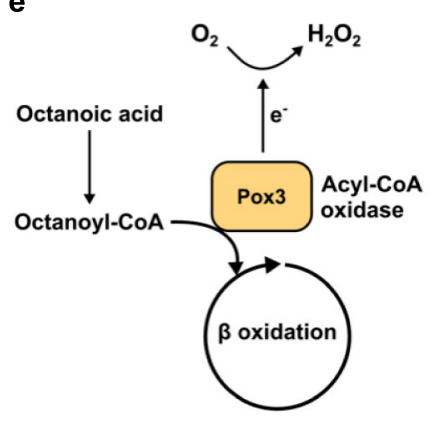

c

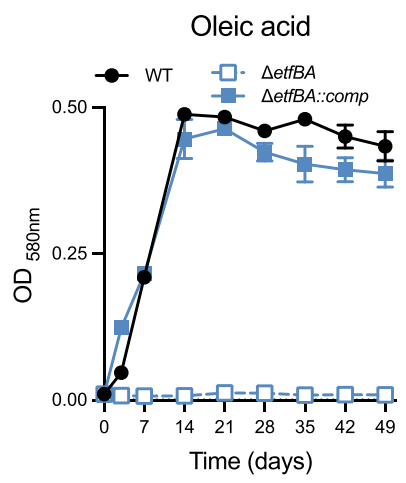

Fig. 6 EtfD and EtfBA constitute a pathway necessary for the activity of acyl-coA dehydrogenases. Growth with glycerol $25 \mathrm{mM}$ (a), butyric acid $2.5 \mathrm{mM}(\mathbf{b})$, oleic acid $250 \mu \mathrm{M}(\mathbf{c})$, and cholesterol $250 \mu \mathrm{M}(\mathbf{d})$ as single carbon sources. Oleic acid was replenished every 3-4 days for the first 14 days of culture to support growth and minimize toxicity. Cholesterol was replenished every to 3-4 days for the first 14 days to minimize precipitation. Data are averages of three replicates and are representative of three independent experiments. Error bars correspond to standard deviation. "Comp" stands for complemented. e Diagram representing acyl-coA oxidase activity on octanoic acid. f Rescue of $\Delta$ etfD and $\Delta$ etfBA growth with $250 \mu M$ octanoic acid as sole carbon source with the expression of the acyl-CoA oxidase Pox3. Octanoic acid was replenished every 3-4 days for the first 14 days of culture to support growth and minimize toxicity. Data are averages of three replicates and are representative of three independent experiments. Error bars correspond to standard deviation. "Comp" stands for complemented.

components of human macrophages ${ }^{31}$ and carbon sources for Mtb. Similarly, cholesterol is a carbon source for Mtb during infection ${ }^{4}$. Hence, the inability to utilize fatty acids and cholesterol, together with the increased susceptibility to long-chain fatty acids likely explain the in vivo essentiality of $\mathrm{EtfD}_{\mathrm{Mtb}}$. The mechanism of long-chain fatty acid toxicity to Mtb is still poorly understood, even though it was first described in the $19400^{\prime} \mathrm{s}^{32}$. In other bacteria, several mechanistic explanations have been proposed for the bactericidal activity of long-chain fatty acids, including membrane potential disruption ${ }^{33,34}$, oxidative stress induction $^{35}$, or fatty acid biosynthesis inhibition ${ }^{36}$. Blocking Mtb cholesterol side chain $\beta$-oxidation was previously shown to impair cholesterol utilization and to result in the accumulation of toxic intermediates, which negatively impacted growth even in the presence of alternative carbon sources ${ }^{7,12}$. We have found that $\Delta$ etfD displays a similar phenotype, further reinforcing a function of EtfD in $\beta$-oxidation.

The metabolome of $\Delta e t f D$ in medium with butyric acid as sole carbon source revealed an accumulation of butyryl-CoA, which indicated an impairment in $\beta$-oxidation. This can explain the inability of $\Delta e t f D$ to oxidize fatty acids and connects $\mathrm{EtfD}_{\mathrm{Mtb}}$ with the $\beta$-oxidation enzymes that act on acyl-CoAs-the ACADs. None of Mtb's thirty-five annotated ACADs are essential in vivo ${ }^{37}$ and there are no reports showing a specific ACAD being essential in vitro for the utilization of any fatty acid. Thus, ACADs are likely to be functionally redundant. However, this redundancy was not sufficient to support growth of $\Delta$ etfD in media with fatty acids as single carbon sources, strongly suggesting that $\mathrm{EtfD}_{\mathrm{Mtb}}$ is necessary for the activity of multiple, if not all, ACADs.

Immunoprecipitation using $\mathrm{EtfD}_{\mathrm{Mtb}}$ as bait revealed multiple possible interacting proteins, suggesting that $\mathrm{EtfD}_{\mathrm{Mtb}}$ might integrate several pathways in Mtb. We were especially interested in the interaction with the cytoplasmic protein $\mathrm{EtfB}_{\mathrm{Mtb}}$, which together with $\mathrm{EtfA}_{\mathrm{Mtb}}$ constitute a putative electron transfer flavoprotein (ETF). Based on the data available for EtfB and EtfA homolog $s^{38}$, these two proteins form a heterodimer. The fact that only EtfB was identified in the co-immunoprecipitation assay suggests that EtfD interacts more strongly with this subunit of Mtb's ETF. The human homologs (EtfAB) form an enzyme that re-oxidizes the FAD co-factor of multiple ACADs and transfers the electrons to the membrane bound oxidoreductase electron transfer flavoprotein oxidoreductase (EtfD), which then reduces the electron carrier ubiquinone, hence contributing to the generation of energy ${ }^{39}$. Mutations rendering defects in EtfAB or EtfD lead to a metabolic disease named multiple acyl-CoA dehydrogenase deficiency, which among other outcomes is characterized by the inability to oxidize fatty acids ${ }^{40}$. This led us to hypothesize that Mtb's EtfBA $A_{M t b}$ and EtfD ${ }_{M t b}$ might work together in a similar pathway. That $\mathrm{EtfD}_{\mathrm{Mtb}}$ and $\mathrm{EtfBA}_{\mathrm{Mtb}}$ show a strong pattern of co-occurrence across bacterial proteomes and $\triangle e t f B A$ was unable to utilize fatty acids and cholesterol as single carbon sources were strong indications in favor of our hypothesis. Nevertheless, for a yet unclear mechanism, it was notable that $\triangle \operatorname{etfBA}$ grew more slowly than $\triangle \operatorname{etf} D$. The expression of the acylCoA oxidase Pox $3^{28,29}$, an enzyme that catalyzes the same 
reaction as ACADs, but uses molecular oxygen as an electron acceptor $^{27}$, was able to rescue the growth of both $\triangle$ etfD and $\triangle$ etfBA in fatty acids as single carbon source, showing that in both cases $\beta$-oxidation was impaired at the ACAD step and confirming our proposed hypothesis. Although we do not have direct evidence, it is very likely that the same phenomenon explains the inability of $\triangle e t f D$ and $\triangle e t f B A$ to utilize long chain fatty acids and cholesterol. Additionally, both mutants, with $\triangle \operatorname{etf} B A$ displaying a stronger phenotype, presented a growth defect even with glycerol as single carbon source. It is possible that defective $\beta$-oxidation might interfere, for example, with mycolic acid recycling ${ }^{41}$. Also, ACADs participate in other metabolic pathways such as branched-chain amino acids catabolism ${ }^{42}$, which might also have contributed to the growth defect in glycerol.

We are reporting a function for EtfD/EtfBA in $\beta$-oxidation, but it is possible that these proteins may have additional roles in $\mathrm{Mtb}$. It has recently been reported that both $\mathrm{EtfBA}_{\mathrm{Mtb}}$ and $\mathrm{EtfD}_{\mathrm{Mtb}}$ are essential for resisting toxicity in media containing heme ${ }^{43}$. This further strengthened the functional relation between these proteins and suggested a role in iron metabolism. Interestingly, transcript levels of etf $D_{\mathrm{Mtb}}$, but not etfBA $A_{\mathrm{Mtb}}$, respond to the iron content of the medium in an IdeR (iron metabolism transcriptional regulator) dependent manner ${ }^{44,45}$. It is also noteworthy, that the investigation of the Mtb transcriptional response to a series of compounds that inhibit $\mathrm{EtfD}^{46}$ suggest a role in redox sensing. Curiously, it was shown that transcription of etfD is repressed by hydrogen peroxide ${ }^{47}$. It is thus possible that EtfD might also act as a redox sensor. Whether $\mathrm{EtfBA}_{\mathrm{Mtb}}$ and/or $\mathrm{EtfD}_{\mathrm{Mtb}}$ complex have a direct or indirect role in heme utilization and redox homeostasis is a question worthwhile to be addressed in the future.

In conclusion, we have identified a complex composed of EtfBA $_{\mathrm{Mtb}}$ and the cognate membrane oxidoreductase $\mathrm{EtfD}_{\mathrm{Mtb}}$ that is required for the function of multiple ACADs. This complex constitutes a previously unsuspected vulnerable component of Mtb's $\beta$-oxidation machinery. EtfD ${ }_{\mathrm{Mtb}}$ has no structural homologs in humans and was recently proposed as the target of a series of compounds that are bactericidal against $\mathrm{Mtb}^{46}$, which suggests that it could be a potential target for TB drug development. The presence of $\mathrm{EtfD}_{\mathrm{Mtb}}$ and $\mathrm{EtfBA}_{\mathrm{Mtb}}$ homologs in Leptospira interrogans suggests that this pathway might be relevant for other pathogens that rely on host fatty acids as carbon sources ${ }^{26}$.

\section{Methods}

Culture conditions. For cloning purposes we used Escherichia coli as a host, which was cultured in LB medium at $37^{\circ} \mathrm{C}$. Mtb was cultured at $37^{\circ} \mathrm{C}$ in different media: Middlebrook $7 \mathrm{H} 9$ supplemented with $0.2 \%$ glycerol, $0.05 \%$ tyloxapol, and $\mathrm{ADNaCl}$ $(0.5 \%$ fatty acid free BSA from Roche, $0.2 \%$ dextrose and $0.85 \% \mathrm{NaCl}$ ) or in Middlebrook $7 \mathrm{H} 10$ supplemented with $0.5 \%$ glycerol and $10 \%$ oleic acid-albumindextrose-catalase (OADC), and in a modified Sauton's minimal medium $(0.05 \%$ potassium dihydrogen phosphate, $0.05 \%$ magnesium sulfate heptahydrate, $0.2 \%$ citric acid, $0.005 \%$ ferric ammonium citrate, and $0.0001 \%$ zinc sulfate) supplemented with $0.05 \%$ tyloxapol, $0.4 \%$ glucose, $0.2 \%$ glycerol, and $\mathrm{ADNaCl}$ with fatty-acid-free $\mathrm{BSA}$ (Roche). Modified Sauton's solid medium contained 1.5\% bactoagar (BD) and glycerol at a higher concentration $(0.5 \%)$. For single or mixed carbon source cultures we have used glycerol $25 \mathrm{mM}$, sodium acetate $2.5 \mathrm{mM}$, propionic acid $2.5 \mathrm{mM}$ and butyric acid $2.5 \mathrm{mM}$. Octanoic acid, palmitic acid, oleic acid, and cholesterol were dissolved in a solution of tyloxapol and ethanol (1:1) and added to the medium at a final concentration of $250 \mu \mathrm{M}$ to modified Sauton's minimal medium with $0.5 \%$ fatty acid free BSA (Roche) and $0.85 \% \mathrm{NaCl}$. Since fatty acids of eight carbons or more are toxic in the $\mathrm{mM}$ range, cultures were replenished every 3-4 days for the first 14 days, as described elsewhere ${ }^{48}$. We also used this strategy for cholesterol medium, due to its poor solubility. Tyloxapol and ethanol (1:1) was also added to the cultures with glycerol, acetic acid, propionic acid and butyric acid in the same quantities, to control for a possible impact on Mtb's growth. Mycobacterium smegmatis $\mathrm{MC}^{2} 155$ was cultured in Middlebrook $7 \mathrm{H} 10$ supplemented with $0.2 \%$ glycerol at $37^{\circ} \mathrm{C}$. Antibiotics were used at the following final concentrations: carbenicillin $100 \mu \mathrm{g} / \mathrm{ml}$, hygromycin 50 $\mu \mathrm{g} / \mathrm{ml}$ and kanamycin $50 \mu \mathrm{g} / \mathrm{ml}$.

Mutant construction. Mtb $\mathrm{H} 37 \mathrm{Rv}$ etf $D_{\mathrm{Mtb}}$ conditional knockdown was generated using a previously described strategy that control expression through proteolysis ${ }^{49}$.
Briefly, a Flag tag and DAS +4 tag were added to the $3^{\prime}$ end of etf $D_{\mathrm{Mtb}}$, at the $3^{\prime}$ end of the target gene. This strain was then transformed with a plasmid expressing the adapter protein $\mathrm{SspB}$ under the control of TetR regulated promoters. In the presence of anhydrotetracycline (ATC) $500 \mu \mathrm{g} / \mathrm{ml}$, TetR loses affinity to the promoter and $s s p B$ expression is de-repressed. SspB acts by delivering DAS + 4-tagged proteins to the native ClpXP protease. Hence, when ATC is added to the culture, $\mathrm{SspB}$ expression is induced and $\mathrm{EtfD}_{\mathrm{Mtb}}$-DAS-tag is degraded, working as a TetOFF system (EtfD $\mathrm{Mtb}_{\mathrm{t}}$-TetOFF).

We have obtained deletion mutants through recombineering by using Mtb $\mathrm{H} 37 \mathrm{Rv}$ expressing the recombinase RecET. Constructs with the hygromycin resistant gene (hygR) flanked by $500 \mathrm{bp}$ upstream and downstream of the target loci were synthesized (GeneScript). In the case of etfD, since the flanking gene aspC is in the same orientation and it is essential for growth ${ }^{50}$, we have included in the construct the constitutive promoter hsp60 to avoid polar effects. Mutants were selected in modified Sauton's with hygromycin. The plasmid expressing recET was counterselected by growing the deleted mutants in modified Sauton's

supplemented with sucrose $10 \%$. For complementation we have cloned etfD and etfBA under the control of the promoter phsp60 into a plasmid with a kanamycin resistant cassette that integrates at the att-L5 site (pMCK-phsp60-etfD and pMCKphsp60-etfBA) and transformed the deleted mutants. The gene pox3 from Yarrowia lipolytica was codon adapted for $M t b$ use, synthesized (GeneScript), cloned into a plasmid under the control of the promoter pTB38, with a kanamycin resistant cassette that integrates in the att-L5 site (pMCK-pTB38-pox3) and transformed into both etfD $D_{\mathrm{Mtb}}$ and etfBA $A_{\mathrm{Mtb}}$ deletion mutants. All generated strains and plasmids are listed in Supplementary Tables 3 and 4, respectively.

Whole genome sequencing. The genetic identity of $\triangle e t f D$ and $\triangle e t f B A$ was confirmed by whole genome sequencing (WGS).

Between 150 and $200 \mathrm{ng}$ of genomic DNA was sheared acoustically and HiSeq sequencing libraries were prepared using the KAPA Hyper Prep Kit (Roche). PCR amplification of the libraries was carried out for ten cycles. 5-10 $\times 10^{6} 50$-bp paired-end reads were obtained for each sample on an Illumina HiSeq 2500 using the TruSeq SBS Kit v3 (Illumina). Post-run demultiplexing and adapter removal were performed and fastq files were inspected using fastqc ${ }^{51}$. Trimmed fastq files were then aligned to the reference genome (M. tuberculosis $\mathrm{H} 37 \mathrm{RvCO}$;

NZ_CM001515.1) using bwa mem47. Bam files were sorted and merged using samtools48. Read groups were added and bam files de-duplicated using Picard tools and GATK best-practices were followed for SNP and indel detection49. Gene knockouts and cassette insertions were verified for all strains by direct comparison of reads spanning insertion points to plasmid maps and the genome sequence. Reads coverage data was obtained from the software Integrative Genomics Viewer version $2.5 .2(\mathrm{IGV})^{52-54}$.

PhoA fusion assay. Truncated versions of $\mathrm{EtfD}_{\mathrm{Mtb}}$ were fused with the $E$. coli alkalyne phosphatase PhoA. This enzyme requires the oxidative environment of the periplasm to be active and degrades the substrate BCIP generating a blue precipitate. We have fused $p h o A$ at different residues located in the transmembrane domains. Positive control consisted in PhoA fused with the antigen $85 \mathrm{~B}$, while the negative control was PhoA alone ${ }^{55}$. All plasmids were transformed in M. smegmatis $\mathrm{MC}^{2} 155$ and the assay was performed in LB plates with and without BCIP.

Mouse infection. Mouse experiments were performed in accordance with the Guide for the Care and Use of Laboratory Animals of the National Institutes of Health, with approval from the Institutional Animal Care and Use Committee of Weill Cornell Medicine. Forty-eight female, eight-week-old Mus musculus C57BL/6 (Jackson Labs) were infected with $~ 100-200 \mathrm{CFU} /$ mouse using an Inhalation Exposure System (Glas-Col). Strains were grown to mid-exponential phase and single-cell suspensions were prepared in PBS with 0.05\% Tween 80, and then resuspended in PBS. Lungs and spleen were homogenized in PBS and plated on modified Sauton's medium to determine CFU/organ at the indicated time points.

Metabolomics. Strains were grown in modified Sauton's until an $\mathrm{OD}_{580 \mathrm{~nm}}$ of 1 and $1 \mathrm{ml}$ of culture was used to seed filters ${ }^{56}$ placed on top of solid modified Sauton's medium. Bacteria grew for 7 days, after which the filters were transferred to solid modified Sauton's medium with butyric acid or ${ }^{13} \mathrm{C}$-labelled butyric acid (Cambridge Isotope Laboratories, Inc) at a final concentration of $2.5 \mathrm{mM}$ for $24 \mathrm{~h}$. For metabolite extraction bacteria were disrupted by bead beating three cycles, $50 \mathrm{~s}$ (Precellys 24, Bertin technologies) in a solution of acetonitrile:methanol:water (4:4:2). The relative abundances of butyric acid, CoA species and TCA intermediates were determined using an ion-pairing LC-MS system, as previously described ${ }^{57}$. In brief, samples $(5 \mathrm{uL})$ were injected onto a ZORBAX RRHD ExtendC18 column $(2.1 \times 150 \mathrm{~mm}, 1.8 \mu \mathrm{m}$; Agilent Technologies $)$ with a ZORBAX SB-C8 $\left(2.1 \mathrm{~mm} \times 30 \mathrm{~mm}, 3.5 \mu \mathrm{m}\right.$; Agilent Technologies) precolumn heated to $40^{\circ} \mathrm{C}$ and separated using a gradient of methanol in $5 \mathrm{mM}$ tributylamine $/ 5.5 \mathrm{mM}$ acetate. Post-column, $10 \%$ dimethyl sulfoxide in acetone $(0.2 \mathrm{ml} / \mathrm{min})$ was mixed with the mobile phases to increase sensitivity. Detection was performed from $\mathrm{m} / z$ 50-1100, using an Agilent Accurate Mass 6230 Time of Flight (TOF) spectrometer with Agilent Jet Stream electrospray ionization source operating in the negative 
ionization mode. Incorporation of ${ }^{13} \mathrm{C}$ was quantitated and corrected for natural ${ }^{13} \mathrm{C}$ abundance using Profinder B.08.00 (Agilent Technologies).

Immunoprecipitation. We transformed $\Delta e t f D$ with a plasmid expressing flag tagged EtfD under hsp60 promoter (pMEK-Phsp60-etfD Mtb -flag) and used WT Mtb expressing only the flag tag as a control. Mtb whole-cell lysates were collected from $120 \mathrm{ml} \log$ phase culture in butyric acid single carbon source Sauton's medium, incubated with $1 \%$ DDM for $2 \mathrm{~h}$ on ice, followed by anti-Flag beads (Sigma) overnight incubation with gentle rotation. Beads were collected on the second day, washed with lysis buffer (50 mM Tris- $\mathrm{HCl}, 50 \mathrm{mM} \mathrm{NaCl}, \mathrm{pH} 7.4$ ), and eluted with $100 \mathrm{ng} / \mu \mathrm{l} \mathrm{Flag}$ peptide. The eluates were resolved on SDS-PAGE before mass spectrometry.

For mass spectrometry analysis, the total spectrum count (TSC) from biological duplicates were summed. We calculated the ratio of summed TSC from EtfD $\mathrm{Mtb}^{-}$ Flag vs. Flag control and used a cut-off of $\geq 10$.

Acyl-CoA oxidase activity in cell lysates. Mtb strains were grown in modified Sauton's minimal medium until mid-exponential phase (OD 0.5). Bacteria were lysed by bead beating three cycles, 50 s (Precellys 24, Bertia technologies) in $11 \mathrm{mM}$ potassium phosphate buffer ( $\mathrm{pH} 7.4)$ with protease inhibitor (cOmplete ${ }^{\mathrm{Tm}}$, Mini Protease Inhibitor Cocktail; Roche). Samples were concentrated with Pierce Protein Concentrators PES, 3K MWCO (ThermoFisher Scientific) and resuspend in the same buffer to exclude metabolites that could interfere with the reaction. The acyl-CoA oxidase assay was adapted from previous reports ${ }^{58,59}$. Briefly, the assay mixture contained $11 \mathrm{mM}$ potassium phosphate buffer ( $\mathrm{pH} 7.4$ ), $40 \mathrm{mM}$ aminotriazole (Sigma-Aldrich), $0.04 \mathrm{mg} / \mathrm{ml}$ peroxidase from horseradish Type VI-A (Sigma-Aldrich), $104 \mu \mathrm{M} 22^{\prime}, 7^{\prime}$-dichlorofluorescin diacetate (Sigma-Aldrich) and 30 $\mu \mathrm{M}$ octanoyl coenzyme A lithium salt hydrate (Sigma-Aldrich). The assays were performed in black 96 well-plates with clear bottom (Costar) with a sample volume of $100 \mu \mathrm{l}$ and a total volume of $200 \mu \mathrm{l}$. Fluorescence (excitation, $503 \mathrm{~nm}$; emission, $529 \mathrm{~nm}$ ) was recorded for $10 \mathrm{~min}$, and the activity was expressed as $2^{\prime}, 7^{\prime}$-dichlorofluorescein (DCF) nmol produced/min $\mathrm{mg}$ of protein. For quantification, we generated a standard curve for DCF (Sigma-Aldrich) dissolved $11 \mathrm{mM}$ potassium phosphate buffer ( $\mathrm{pH}$ 7.4). Protein was quantified using the Qubit ${ }^{\mathrm{ru}}$ Protein Assay Kit (ThermoFisher Scientific).

In silico analysis. Transmembrane domain topology of $\mathrm{EtfD}_{\mathrm{Mtb}}$ was performed in MEMSAT3 $^{21}$. Domain architecture of EtfD ${ }_{M t b}$, EtfB and EtfA was based on HHPred $^{60}$ and XtalPred-RF ${ }^{61}$. Eggnog ${ }^{62}$ was used for the cluster of orthologous groups analysis (COG). The members of COG247 that include etfD were used to generate a rootless phylogenetic tree in iTOL (version 6$)^{63}$.

To analyze the presence or absence of $\mathrm{EtfD}_{\mathrm{Mtb}}, \mathrm{EtfB}_{\mathrm{Mtb}}$, and $\mathrm{EtfA}_{\mathrm{Mtb}}$ homologs across bacterial species, we obtained the set of UniProt reference bacterial proteomes, which are are selected both manually and algorithmically by UniProt as landmarks in (bacterial) proteome space ${ }^{64}$. We discarded proteomes with no taxonomic labels and performed the analysis on a final set of 6240 bacterial reference proteomes. Using $\mathrm{EtfD}_{\mathrm{Mtb}}, \mathrm{EtfB}_{\mathrm{Mtb}}$, and $\mathrm{EtfA}_{\mathrm{Mtb}}$ as query protein sequences, we used the following protein BLAST (BLASTp) parameter values: identity cutoff of $>30 \%$, coverage cutoff of $>75 \%$, $e$-value cutoff of $10-3$. Visual representations of phylogenies with surrounding color-coded rings were generated using the software tool GraPhLan ${ }^{65}$, with the phylogenetic try built from the taxonomic categorization of the 6240 UniProt bacterial reference proteomes. To evaluate the statistical significance of co-occurrence of EtfD $\mathrm{Mtb}_{\mathrm{tb}}$ and EtfBA $\mathrm{A}_{\mathrm{Mtb}}$ in Actinobacteria, we performed a hypergeometric test to evaluate the probability of observing $\mathrm{k}$ species with EtfD and EtfBA homologs, given $\mathrm{M}$ Actinobacterial species, $\mathrm{n}$ species with EtfD homologs, and $\mathrm{N}$ species with EtfBA homologs.

Quantification and statistical analysis. Generation of graphics and data analyses were performed in Prism version 9.0 software (GraphPad).

Reporting summary. Further information on research design is available in the Nature Research Reporting Summary linked to this article.

\section{Data availability}

Whole genome sequencing data generated in this study were deposited in NCBI's Sequence Read Archive (SRA) under BioProject PRJNA670664. LC-MS data generated in this study were deposited in the MetaboLights database ${ }^{66}$ under accession code MTBLS2374. To identify possible homologues of EtfD, EtfB, and EtfA, we used the subset of Uniprot reference proteomes corresponding to bacteria (https://www.uniprot.org/ proteomes/?query $=* \&$ fil $=$ taxonomy $\% 3 \mathrm{~A} \% 22 \mathrm{Bacteria}+\% 5 \mathrm{~B} 2 \% 5 \mathrm{D} \% 22+\mathrm{AND}$ + reference\%3Ayes). Source data for figures are provided as a Source Data file. Source data are provided with this paper.

Received: 10 February 2021; Accepted: 26 October 2021; Published online: 15 November 2021

\section{References}

1. Rao, S. P., Alonso, S., Rand, L., Dick, T. \& Pethe, K. The protonmotive force is required for maintaining ATP homeostasis and viability of hypoxic, nonreplicating Mycobacterium tuberculosis. Proc. Natl Acad. Sci. USA 105, 11945-11950 (2008).

2. Mahajan, R. Bedaquiline: first FDA-approved tuberculosis drug in 40 years. Int. J. Appl. Basic Med. Res. 3, 1-2 (2013).

3. Nazarova, E. V. et al. Rv3723/LucA coordinates fatty acid and cholesterol uptake in Mycobacterium tuberculosis. Elife 6, e26969 (2017).

4. Pandey, A. K. \& Sassetti, C. M. Mycobacterial persistence requires the utilization of host cholesterol. Proc. Natl Acad. Sci. USA 105, 4376-4380 (2008).

5. Gioffré, A. et al. Mutation in mce operons attenuates Mycobacterium tuberculosis virulence. Microbes Infect. 7, 325-334 (2005).

6. Shimono, N. et al. Hypervirulent mutant of Mycobacterium tuberculosis resulting from disruption of the mcel operon. Proc. Natl Acad. Sci. USA 100, 15918-15923 (2003).

7. Thomas, S. T., VanderVen, B. C., Sherman, D. R., Russell, D. G. \& Sampson, N. S. Pathway profiling in Mycobacterium tuberculosis: elucidation of cholesterol-derived catabolite and enzymes that catalyze its metabolism. J. Biol. Chem. 286, 43668-43678 (2011).

8. Thomas, S. T. \& Sampson, N. S. Mycobacterium tuberculosis utilizes a unique heterotetrameric structure for dehydrogenation of the cholesterol side chain. Biochemistry 52, 2895-2904 (2013).

9. Yang, M., Guja, K. E., Thomas, S. T., Garcia-Diaz, M. \& Sampson, N. S. A distinct MaoC-like enoyl-CoA hydratase architecture mediates cholesterol catabolism in Mycobacterium tuberculosis. ACS Chem. Biol. 9, 2632-2645 (2014).

10. Carere, J., McKenna, S. E., Kimber, M. S. \& Seah, S. Y. Characterization of an aldolase-dehydrogenase complex from the cholesterol degradation pathway of Mycobacterium tuberculosis. Biochemistry 52, 3502-3511 (2013).

11. Crowe, A. M. et al. Catabolism of the last two steroid rings in Mycobacterium tuberculosis and other bacteria. mBio 8, e00321-17 (2017).

12. Chang, J. C. et al. igr Genes and Mycobacterium tuberculosis cholesterol metabolism. J. Bacteriol. 191, 5232-5239 (2009).

13. $\mathrm{Hu}, \mathrm{Y}$. et al. 3-Ketosteroid 9alpha-hydroxylase is an essential factor in the pathogenesis of Mycobacterium tuberculosis. Mol. Microbiol. 75, 107-121 (2010).

14. Nesbitt, N. M. et al. A thiolase of Mycobacterium tuberculosis is required for virulence and production of androstenedione and androstadienedione from cholesterol. Infect. Immun. 78, 275-282 (2010).

15. VanderVen, B. C. et al. Novel inhibitors of cholesterol degradation in Mycobacterium tuberculosis reveal how the bacterium's metabolism is constrained by the intracellular environment. PLoS Pathog. 11, e1004679 (2015).

16. Cole, S. T. et al. Deciphering the biology of Mycobacterium tuberculosis from the complete genome sequence. Nature 393, 537-544 (1998).

17. Cox, J. S., Chen, B., McNeil, M. \& Jacobs, W. R. Jr. Complex lipid determines tissue-specific replication of Mycobacterium tuberculosis in mice. Nature 402, 79-83 (1999).

18. Wipperman, M. F., Yang, M., Thomas, S. T. \& Sampson, N. S. Shrinking the FadE proteome of Mycobacterium tuberculosis: insights into cholesterol metabolism through identification of an $\alpha 2 \beta 2$ heterotetrameric acyl coenzyme A dehydrogenase family. J. Bacteriol. 195, 4331-4341 (2013).

19. Sassetti, C. M., Boyd, D. H. \& Rubin, E. J. Genes required for mycobacterial growth defined by high density mutagenesis. Mol. Microbiol. 48, 77-84 (2003).

20. DeJesus, M. A. et al. Comprehensive essentiality analysis of the Mycobacterium tuberculosis genome via saturating transposon mutagenesis. mBio 8, e02133-16 (2017).

21. Jones, D. T. Improving the accuracy of transmembrane protein topology prediction using evolutionary information. Bioinformatics 23, 538-544 (2007).

22. Schnappinger, D., O’Brien, K. M. \& Ehrt, S. Construction of conditional knockdown mutants in mycobacteria. Methods Mol. Biol. 1285, 151-175 (2015).

23. Beites, T. et al. Plasticity of the Mycobacterium tuberculosis respiratory chain and its impact on tuberculosis drug development. Nat. Commun. 10, 4970 (2019).

24. Sato, K., Nishina, Y. \& Shiga, K. Electron-transferring flavoprotein has an AMP-binding site in addition to the FAD-binding site. J. Biochem. 114, 215-222 (1993).

25. Ruzicka, F. J. \& Beinert, H. A new iron-sulfur flavoprotein of the respiratory chain. A component of the fatty acid beta oxidation pathway. J. Biol. Chem. 252, 8440-8445 (1977).

26. Henneberry, R. C. \& Cox, C. D. Beta-oxidation of fatty acids by Leptospira. Can. J. Microbiol. 16, 41-45 (1970).

27. Nakajima, Y. et al. Three-dimensional structure of the flavoenzyme acyl-CoA oxidase-II from rat liver, the peroxisomal counterpart of mitochondrial acylCoA dehydrogenase. J. Biochem. 131, 365-374 (2002).

28. Wang, H. et al. Cloning and characterization of the peroxisomal acyl CoA oxidase ACO3 gene from the alkane-utilizing yeast Yarrowia lipolytica. Yeast 14, 1373-1386 (1998).

29. Wang, H. J. et al. Evaluation of acyl coenzyme A oxidase (Aox) isozyme function in the n-alkane-assimilating yeast Yarrowia lipolytica. J. Bacteriol. 181, 5140-5148 (1999). 
30. Cook, G. M., Greening, C., Hards, K. \& Berney, M. Energetics of pathogenic bacteria and opportunities for drug development. Adv. Microb. Physiol. 65, 1-62 (2014).

31. Mahoney, E. M., Scott, W. A., Landsberger, F. R., Hamill, A. L. \& Cohn, Z. A. Influence of fatty acyl substitution on the composition and function of macrophage membranes. J. Biol. Chem. 255, 4910-4917 (1980).

32. Dubos, R. J. The effect of lipids and serum albumin on bacterial growth. J. Exp. Med. 85, 9-22 (1947).

33. Galbraith, H. \& Miller, T. B. Effect of long chain fatty acids on bacterial respiration and amino acid uptake. J. Appl. Bacteriol. 36, 659-675 (1973).

34. Greenway, D. L. \& Dyke, K. G. Mechanism of the inhibitory action of linoleic acid on the growth of Staphylococcus aureus. J. Gen. Microbiol. 115, 233-245 (1979).

35. Doi, H., Hoshino, Y., Nakase, K. \& Usuda, Y. Reduction of hydrogen peroxide stress derived from fatty acid beta-oxidation improves fatty acid utilization in Escherichia coli. Appl. Microbiol. Biotechnol. 98, 629-639 (2014).

36. Zheng, C. J. et al. Fatty acid synthesis is a target for antibacterial activity of unsaturated fatty acids. FEBS Lett. 579, 5157-5162 (2005).

37. Zhang, Y. J. et al. Tryptophan biosynthesis protects mycobacteria from CD4 T-cell-mediated killing. Cell 155, 1296-1308 (2013).

38. Roberts, D. L., Frerman, F. E. \& Kim, J. J. Three-dimensional structure of human electron transfer flavoprotein to 2.1-A resolution. Proc. Natl Acad. Sci. USA 93, 14355-14360 (1996)

39. Watmough, N. J. \& Frerman, F. E. The electron transfer flavoprotein: ubiquinone oxidoreductases. Biochim. Biophys. Acta 1797, 1910-1916 (2010).

40. Olsen, R. K. et al. Clear relationship between ETF/ETFDH genotype and phenotype in patients with multiple acyl-CoA dehydrogenation deficiency. Hum. Mutat. 22, 12-23 (2003)

41. Dunphy, K. Y., Senaratne, R. H., Masuzawa, M., Kendall, L. V. \& Riley, L. W. Attenuation of Mycobacterium tuberculosis functionally disrupted in a fatty acyl-coenzyme A synthetase gene fadD5. J. Infect. Dis. 201, 1232-1239 (2010).

42. Rani, N., Hazra, S., Singh, A. \& Surolia, A. Functional annotation of putative fadE9 of Mycobacterium tuberculosis as isobutyryl-CoA dehydrogenase involved in valine catabolism. Int. J. Biol. Macromol. 122, 45-57 (2019).

43. Zhang, L. et al. Comprehensive analysis of iron utilization by Mycobacterium tuberculosis. PLoS Pathog. 16, e1008337 (2020).

44. Rodriguez, G. M., Voskuil, M. I., Gold, B., Schoolnik, G. K. \& Smith, I. ideR, An essential gene in Mycobacterium tuberculosis: role of IdeR in irondependent gene expression, iron metabolism, and oxidative stress response. Infect. Immun. 70, 3371-3381 (2002).

45. Gold, B., Rodriguez, G. M., Marras, S. A., Pentecost, M. \& Smith, I. The Mycobacterium tuberculosis IdeR is a dual functional regulator that controls transcription of genes involved in iron acquisition, iron storage and survival in macrophages. Mol. Microbiol. 42, 851-865 (2001).

46. Székely, R. et al. 6,11-Dioxobenzo[f]pyrido[1,2-a]indoles kill Mycobacterium tuberculosis by targeting iron-sulfur protein Rv0338c (IspQ), a putative redox sensor. ACS Infect. Dis. 6, 3015-3025 (2020).

47. Schnappinger, D. et al. Transcriptional adaptation of Mycobacterium tuberculosis within macrophages: insights into the phagosomal environment. J. Exp. Med. 198, 693-704 (2003).

48. Gouzy, A., Healy, C., Black, K. A., Rhee, K. Y. \& Ehrt, S. Growth of Mycobacterium tuberculosis at acidic $\mathrm{pH}$ depends on lipid assimilation and is accompanied by reduced GAPDH activity. Proc. Natl. Acad. Sci. USA 118, e2024571118 (2021)

49. Kim, J. H. et al. Protein inactivation in mycobacteria by controlled proteolysis and its application to deplete the beta subunit of RNA polymerase. Nucleic Acids Res. 39, 2210-2220 (2011).

50. Jansen, R. S. et al. Aspartate aminotransferase Rv3722c governs aspartatedependent nitrogen metabolism in Mycobacterium tuberculosis. Nat. Commun. 11, 1960 (2020).

51. Andrews, S. FastQC: a quality control tool for high throughput sequence data. http://www.bioinformatics.babraham.ac.uk/projects/fastqc (2010).

52. Robinson, J. T. et al. Integrative genomics viewer. Nat. Biotechnol. 29, 24 (2011).

53. Thorvaldsdottir, H., Robinson, J. T. \& Mesirov, J. P. Integrative Genomics Viewer (IGV): high-performance genomics data visualization and exploration. Brief Bioinform. 14, 178-192 (2013).

54. Robinson, J. T., Thorvaldsdottir, H., Wenger, A. M., Zehir, A. \& Mesirov, J. P. Variant review with the integrative genomics viewer. Cancer Res. 77, e31-e34 (2017).

55. Braunstein, M. et al. Identification of genes encoding exported Mycobacterium tuberculosis proteins using a Tn552'phoA in vitro transposition system. $J$. Bacteriol. 182, 2732-2740 (2000).

56. Nandakumar, M., Prosser, G. A., de Carvalho, L. P. \& Rhee, K. Metabolomics of Mycobacterium tuberculosis. Methods Mol. Biol. 1285, 105-115 (2015)
57. Goncalves, M. D. et al. High-fructose corn syrup enhances intestinal tumor growth in mice. Science 363, 1345-1349 (2019).

58. Small, G. M., Burdett, K. \& Connock, M. J. A sensitive spectrophotometric assay for peroxisomal acyl-CoA oxidase. Biochem. J. 227, 205-210 (1985)

59. Taguchi, H., Ogura, Y., Takanashi, T., Hashizoe, M. \& Honda, Y. In vivo quantitation of peroxides in the vitreous humor by fluorophotometry. Invest. Ophthalmol. Vis. Sci. 37, 1444-1450 (1996).

60. Zimmermann, L. et al. A completely reimplemented MPI Bioinformatics Toolkit with a new HHpred server at its core. J. Mol. Biol. 430, 2237-2243 (2018).

61. Slabinski, L. et al. XtalPred: a web server for prediction of protein crystallizability. Bioinformatics 23, 3403-3405 (2007).

62. Jensen, L. J. et al. eggNOG: automated construction and annotation of orthologous groups of genes. Nucleic Acids Res. 36, D250-D254 (2008).

63. Letunic, I. \& Bork, P. Interactive Tree Of Life (iTOL) v4: recent updates and new developments. Nucleic Acids Res. 47, W256-w259 (2019).

64. UniProt Consortium. UniProt: a worldwide hub of protein knowledge. Nucleic Acids Res. 47, D506-d515 (2019).

65. Asnicar, F., Weingart, G., Tickle, T. L., Huttenhower, C. \& Segata, N. Compact graphical representation of phylogenetic data and metadata with GraPhlAn. PeerJ 3, e1029 (2015)

66. Haug, K. et al. MetaboLights: a resource evolving in response to the needs of its scientific community. Nucleic Acids Res. 48, D440-d444 (2020)

\section{Acknowledgements}

We thank J. McConnell and C. Trujillo for help with strain generation and in vivo characterization. We thank K.G. Papavinasasundaram (University of Massachusetts), R. Aslebagh and S.A. Shaffer (University of Massachusetts, Mass Spectrometry Facility) for LC-MS/MS analysis. We acknowledge J.M. Bean from MSKCC and the use of the Integrated Genomics Operation Core at MSKCC, funded by the NCI Cancer Center Support Grant (CCSG, P30 CA08748), Cycle for Survival and the Marie-Josée and Henry R. Kravis Center for Molecular Oncology. D.S. and S.E. were supported by Tri-Institutional TB Research Unit U19AI111143 T.B. was supported by a Potts Memorial Foundation fellowship.

\section{Author contributions}

S.E. and D.S. conceived ideas, supervised the study and revised the manuscript. T.B. conceived ideas, performed experimental work, analysed and interpreted data and wrote the manuscript. R.S.J. and R.W. performed experimental work, analysed and interpreted data and reviewed the manuscript. A.J. performed the in silico analysis and reviewed the manuscript. K.Y.R. supervised the metabolomics experiments and reviewed the manuscript.

\section{Competing interests}

The authors declare no competing interests.

\section{Additional information}

Supplementary information The online version contains supplementary material available at https://doi.org/10.1038/s41467-021-26941-1.

Correspondence and requests for materials should be addressed to Sabine Ehrt.

Peer review information Nature Communications thanks Caroline Demangel, Lindsay Eltis and the other, anonymous, reviewer for their contribution to the peer review of this work. Peer reviewer reports are available.

Reprints and permission information is available at http://www.nature.com/reprints

Publisher's note Springer Nature remains neutral with regard to jurisdictional claims in published maps and institutional affiliations.

Open Access This article is licensed under a Creative Commons Attribution 4.0 International License, which permits use, sharing, adaptation, distribution and reproduction in any medium or format, as long as you give appropriate credit to the original author(s) and the source, provide a link to the Creative Commons license, and indicate if changes were made. The images or other third party material in this article are included in the article's Creative Commons license, unless indicated otherwise in a credit line to the material. If material is not included in the article's Creative Commons license and your intended use is not permitted by statutory regulation or exceeds the permitted use, you will need to obtain permission directly from the copyright holder. To view a copy of this license, visit http://creativecommons.org/ licenses/by/4.0/.

(C) The Author(s) 2021 